J. Kordylewski proved in [3] a theorem about the existence of continous solutions of equation (25), under assumptions (on the function $F^{\prime}$ ) weaker than in the quoted paper [5].

If we shall accept assumptions named in [3], then our theorems will stay true for suitable particular cases of equation (25). Proofs do not change in any essential manner.

\title{
References
}

[1] M. Bajraktarević, Sur une équation fonctionnelle, Glasnik mat. fiz. i astr. 12 (1957), p. 201-205.

[2] M. Ghermăneseu, Sur une classe d'équations fonctionnelles du premier ordre, Acta Math. 75 (1943), p. 191-218.

[3] J. Kordylewski, On the functional equation $F\left(x, p(x), \varphi[f(x)], \varphi\left[f^{2}(x)\right]\right.$, $\left.\ldots, \varphi\left[f^{n}(x)\right]\right)=0$, Ann. Pol. Math. 9 (1961), p. 285-293.

[4] J. Kordylewski and M. Kuczma, On the functional equation $F(x, \varphi(x)$, $\varphi[f(x)])=0$, Ann. Pol. Math. 7 (1959), p. 21-32.

[5] - On the functional equation $F\left(x, \varphi(x), \varphi\left[f_{1}(x)\right], \ldots, \varphi\left[f_{n}(x)\right]\right)=0$, Ann. Pol. Math. 8 (1960), p. 55-60.

[6] M. Kuczma, On continuous solutions of a funotional equation, Ann. Pol. Math. 8 (1960), p. 209-214.

[7] - On the functional equation $\varphi(x)+\varphi[f(x)]=F(x)$, Ann. Pol. Math. 6 (1959), p. $281-287$.

Regu par la Rédaction le 17. 5. 1960

\section{On some extensions of Cauchy's condensation theorem}

\author{
by C. T. RAJAGOPAL (Madras, India)
}

1. Introduction. A. Alexiewicz $([1]$, p. 85) has proved by functional analysis the following theorem, and has shown that the case $b_{n} \equiv 1$ of the theorem at once completes and extends the familiar condensation theorem (test) of Cauchy ([1], p. 80).

THEOREM I. $\left({ }^{1}\right)$ Let $a_{n} \geqslant 0, b_{n} \geqslant 0 \quad(n=1,2, \ldots)$ be given positive sequences and $\left\{\varepsilon_{n}\right\}$ an arbitrary positive sequence tending monotonically to 0 . Then the series

$$
\text { (A) } \quad \sum_{n=1}^{\infty} a_{n} \varepsilon_{n}, \quad \text { (B) } \quad \sum_{n=1}^{\infty} b_{n} \varepsilon_{n}
$$

are either both convergent or both divergent if and only if

$$
0<\varlimsup_{\overline{n \rightarrow \infty}} \frac{b_{1}+b_{2}+\ldots+b_{n}}{a_{1}+a_{2}+\ldots+a_{n}}<\infty,
$$

i.e. the two limits in (1) are both finite and strictly positive.

This note gives a simple proof of Theorem I not depending on functional analysis and deduces from the theorem the following extension of Cauchy's condensation test, due in effect to O. Szasz ([4], p. 1397, Theorem 1).

THEOREM II. Let $\{f(n)\}$ be a positive sequence quasi-monotonic decreasing in the sense that there is an $a \geqslant 0$ such that

$$
f(n+1) \leqslant(1+\alpha / n) f(n) \quad \text { for } \quad n>n_{0}(\alpha) .
$$

Let $\left\{\lambda_{n}\right\}$ be any sequence of positive integers such that

$$
\lambda_{n} \nearrow \infty, \quad \varlimsup_{n \rightarrow \infty} \lambda_{n} / \lambda_{n-1}<\infty .
$$

(1) Added 17th October 1960. After I had sent the MS of this paper to Professor Alexiemicz, I found in the review of his paper [1] by D. Gaier, in Zbl. für Math. 77 (1958), p. 277, a proof of the simpler 'if' or sufficiency part of Theorem I in the case $b_{n} \equiv 1$ which is the same as the proof in this paper. 
Then the series

$$
\sum_{n=1}^{\infty} f(n) \quad \text { and } . \sum_{n=1}^{\infty}\left(\lambda_{n}-\lambda_{n-1}\right) f\left(\lambda_{n}\right) \quad\left(\lambda_{0}=0\right)
$$

are either both convergent or both divergent, the condition on $\lambda_{n} / \lambda_{n-1}$ in (3) being indispensable for this conclusion in its entirety.

The present note also exhibits the results of working with a real sequence instead of the $\left\{b_{n}\right\}$ of Theorem I, in Theorem III which follows and Theorems III', III" of $\S 3$.

THEOREM III. Let $\left\{a_{n}\right\},\left\{u_{n}\right\}$ be sequences of which the first is positive and the second only real. Let $\left\{\varepsilon_{n}\right\}$ be any sequence tending monotonically to 0 . Then the series

$$
\text { (A) } \quad \sum_{n=1}^{\infty} a_{n} \varepsilon_{n}, \quad \text { (U) } \quad \sum_{n=1}^{\infty} u_{n} \varepsilon_{n}
$$

have either both finite sums, or both infinite sums in the sense that the partial sums of each tend to $\pm \infty$, if

$$
\left\{\begin{aligned}
0 & <\varlimsup_{n \rightarrow \infty} \frac{u_{1}+u_{2}+\ldots+u_{n}}{a_{1}+a_{2}+\ldots+a_{n}}<\infty, \\
\text { (or alternatively, }-\infty & \left.<\varlimsup_{n \rightarrow \infty} \frac{u_{1}+u_{2}+\ldots+u_{n}}{a_{1}+a_{2}+\ldots+a_{n}}<0\right) .
\end{aligned}\right.
$$

A consequence of Theorem IIr is the second extension of Cauchy's condensation test given below.

THEOREM IV. Let $\{f(n)\}$ be a positive monotonic decreasing sequence. Let $\left\{\lambda_{n}\right\}$ be a sequence of positive integers satisfying (3) and $\left\{\mu_{n}\right\}$ any real sequence such that

$$
\left\{\begin{aligned}
0 & <\varlimsup_{n \rightarrow \infty} \frac{\mu_{n}}{\lambda_{n}}<\infty \\
(\text { or alternatively, }-\infty & \left.<\varlimsup_{n \rightarrow \infty} \frac{\mu_{n}}{\lambda_{n}}<0\right)
\end{aligned}\right.
$$

Then the series

$$
\sum_{n=1}^{\infty} f(n) \quad \text { and } \quad \sum_{n=1}^{\infty}\left(\mu_{n}-\mu_{n-1}\right) f\left(\lambda_{n}\right) \quad\left(\mu_{0}=0\right)
$$

have either both finite sums or both infinite sums.

2. Lemmas. The proofs of Theorems I, IIr depend on the following simple lemmas established mainly by using Abel's method of partial summation.
LeMrMa 1. If $\left\{\Lambda_{n}\right\}$ is an arbitrary monotonic increasing divergent sequence and $\left\{u_{n}\right\}$ is a sequence, supposed to be real for simplicity, such that $\sum_{n=1}^{\infty} u_{n}$ is convergent, then

$$
\lim _{n \rightarrow \infty}\left(u_{1} \Lambda_{1}+u_{2} \Lambda_{2}+\ldots+u_{n} \Lambda_{n}\right) \Lambda_{n}^{-1}=0 .
$$

This result, together with a converse of it, is well-known (e.g. [3], Corollary of Theorem 1).

LEMma 2. If $\left\{a_{n}\right\},\left\{\varepsilon_{n}\right\}$ are positive sequences and $\varepsilon_{n} \searrow 0$, then the series

$$
\text { (A) } \quad \sum_{n=1}^{\infty} a_{n} \varepsilon_{n}, \quad\left(\mathrm{~A}^{\prime}\right) \quad \sum_{n=1}^{\infty}\left(a_{1}+\ldots+a_{n}\right)\left(\varepsilon_{n}-\varepsilon_{n+1}\right)
$$

are either both convergent or both divergent.

Proof. By partial summation,

(6) $\sum_{r=1}^{n} a_{r} \varepsilon_{r}=\sum_{r=1}^{n-1}\left(a_{1}+\ldots+a_{r}\right)\left(\varepsilon_{r}-\varepsilon_{r+1}\right)+\left(a_{1}+\ldots+a_{n}\right) \varepsilon_{n}, \quad n \geqslant 2$.

If series (A) is convergent, then so is $\left(A^{\prime}\right)$ by the use in (6) of Lemma 1 with $u_{n}=a_{n} \varepsilon_{n}$ and $\Lambda_{n}=1 / \varepsilon_{n}$. On the other hand, if $(\mathrm{A})$ is divergent and $\left(\mathrm{A}^{\prime}\right)$ convergent, then necessarily $\left(a_{1}+\ldots+a_{n}\right) \varepsilon_{n} \rightarrow \infty$, i.e. given any $G>0$, we can find $n_{0}(G)$ so that

or

$$
\left(a_{1}+\ldots+a_{n}\right) \varepsilon_{n}>G \text { for } \quad u>n_{0},
$$

$$
\sum_{r=n_{0}+1}^{N}\left(a_{1}+\ldots+a_{r}\right)\left(\varepsilon_{r}-\varepsilon_{r+1}\right) \geqslant \frac{G}{\varepsilon_{n_{0}+1}} \sum_{r=n_{0}+1}^{N}\left(\varepsilon_{r}-\varepsilon_{r+1}\right)=G\left(1-\frac{\varepsilon_{N+1}}{\varepsilon_{n_{0}+1}}\right) \rightarrow G
$$

as $N \rightarrow \infty$. Thus our assumption, that $\left(\mathrm{A}^{\prime}\right)$ is convergent when (A) is not, leads to a contradiction and so completes the proof.

The proof of the above lemma suffices to establish the following.

LEMara 2a. If $\left\{u_{n}\right\}$ is a real sequence, $\left\{\varepsilon_{n}\right\}$ a positive sequence such that $\varepsilon_{n} \searrow 0$, then the series

$$
\text { (U) } \quad \sum_{n=1}^{\infty} u_{n} \varepsilon_{n}, \quad\left(\mathrm{U}^{\prime}\right) \quad \sum_{n=1}^{\infty}\left(u_{1}+\ldots+u_{n}\right)\left(\varepsilon_{n}-\varepsilon_{n+1}\right)
$$

are so related that the convergence of $(\mathrm{U})$ implies that of $\left(\mathrm{U}^{\prime}\right)$ while the convergence of $\left(U^{\prime}\right)$ implies that of $\left(U^{\prime}\right)$ when $\left(u_{1}+\ldots+u_{n}\right) \varepsilon_{n}$ converges, necessarily to 0 . 
LEMMA 3. If $\left\{a_{n}\right\},\left\{\varepsilon_{n}\right\}$ are positive sequences such that $\varepsilon_{n} \backslash 0, \sum_{n=1}^{\infty} a_{n} \varepsilon_{n}$ converges, and if $\left\{u_{n}\right\}$ is a real sequence such that

$$
\varlimsup_{n \rightarrow \infty} \frac{\left|u_{1}+\ldots+u_{n}\right|}{a_{1}+\ldots+a_{n}}<\infty
$$

then $\sum_{n=1}^{\infty} u_{n} \varepsilon_{n}$ converges.

Lemma 3 is due to Th. Kaluza in the case $a_{n} \equiv 1$ ([3], Satz 20) and follows from Lemmas $2,2 a$. Using the notation of these lemmas, we see that the convergence of series $(A)$ ensures that of $\left(A^{\prime}\right)$, that of: $\left(A^{\prime}\right)$ ensures the absolute convergence of $\left(U^{\prime}\right)$ on account of (7), and finally the convergence of $\left(U^{\prime}\right)$ ensures that of $(U)$ since, by $(7)$ and Lemma 1 ,

$$
\lim _{n \rightarrow \infty}\left(u_{1}+\ldots+u_{n}\right) \varepsilon_{n}=\lim _{n \rightarrow \infty}\left(a_{1}+\ldots+a_{n}\right) \varepsilon_{n}=0 \text {. }
$$

The next lemma contains a result complementary to the preceeding lemma.

LEMCMA 4. If $\left\{a_{n}\right\},\left\{\varepsilon_{n}\right\}$ are positive sequences such that

and $\left\{u_{n}\right\}$ is any real sequence, then

$$
\varepsilon_{n} \searrow 0, \quad \sum_{n=1}^{\infty} a_{n} \varepsilon_{n} \text { diverges }
$$

$$
\varlimsup_{n \rightarrow \infty} \frac{\sum_{r=1}^{n} u_{r} \varepsilon_{r}}{\sum_{r=1}^{n} a_{r} \varepsilon_{r}}\left\{\begin{array}{l}
\leqslant \varlimsup_{n \rightarrow \infty} \frac{u_{1}+\ldots+u_{n}}{a_{1}+\ldots+a_{n}}, \\
\geqslant \varliminf_{n \rightarrow \infty} \frac{u_{1}+\ldots+u_{n}}{a_{1}+\ldots+a_{n}} .
\end{array}\right.
$$

This lemma is one part of a result of mine proved elsewhore ([3], p. 159, Remark (2) following Lemma 1). The part in question is that, if $\left\{M_{n}\right\},\left\{\Lambda_{n}\right\}$ are positive monotonic increasing divergent sequences, and $\left\{U_{n}\right\}$ is any real sequence, then

$$
\varlimsup_{n \rightarrow \infty} \frac{U_{n}}{\Lambda_{n}}\left\{\begin{array}{l}
\leqslant \varlimsup_{n \rightarrow \infty} \frac{\sum_{r=1}^{n}\left(U_{r}-U_{r-1}\right) M_{r}}{\sum_{r=1}^{n}\left(\Lambda_{r}-\Lambda_{r-1}\right) M_{r}} \\
\geqslant \varliminf_{n \rightarrow \infty} \frac{\sum_{r=1}^{n}\left(U_{r}-U_{r-1}\right) M_{r}}{\sum_{r=1}^{n}\left(\Lambda_{r}-\Lambda_{r-1}\right) M_{r}}
\end{array} \quad\left(U_{0}=A_{0}=0\right) .\right.
$$

(8) is simply the above inequality with

$$
M_{n}=\varepsilon_{n}^{-1}, \quad \Lambda_{n}=a_{1} \varepsilon_{1}+\ldots+a_{n} \varepsilon_{n}, \quad U_{n}=u_{1} \varepsilon_{1}+\ldots+u_{n} \varepsilon_{n} .
$$

Before closing this section two remarks may be inserted.
Remark 1. In Lemmas 2-4, and in results based on them, the assumption that $\varepsilon_{n} \searrow 0$ can clearly be replaced by the assumption that $\left\{\varepsilon_{n}\right\}$ is a null sequence which is ultimately monotonic decreasing.

Remark 2. A necessary and sufficient condition for a positive sequence $\{f(n)\}$ to be quasi-monotonic according to (2) with a certain $\alpha \geqslant 0$ is the existence of a $\beta \geqslant 0$ such that

$$
f(n+1) \leqslant(1+1 / n)^{\beta} f(n) \text { for all large } n,
$$

or $\left\{f(n) / n^{\beta}\right\}$ is ultimately monotonic decreasing. In fact, from (2) follows $\left(2^{\prime}\right)$ for any $\beta>\alpha$ and from $\left(2^{\prime}\right)$ follows (2) for any $\alpha>\beta$.

\section{Proofs of Theorems I-IV and supplementary theorems.} Proof of Theorem I. After Lemma 2, we have only to prove that, for the series

$$
\text { (A') } \quad \sum_{n=1}^{\infty}\left(a_{1}+\ldots+a_{n}\right)\left(\varepsilon_{n}-\varepsilon_{n+1}\right), \quad\left(\mathrm{B}^{\prime}\right) \quad \sum_{n=1}^{\infty}\left(b_{1}+\ldots+b_{n}\right)\left(\varepsilon_{n}-\varepsilon_{n+1}\right)
$$

to be either both convergent or both divergent, with every sequence $\left\{\varepsilon_{n}\right\}$ as specified in the theorem, condition (1) is both necessary and sufficient.

The proof of sufficiency is obvious. To prove necessity, we may show as below that, given positive sequences $\left\{a_{n}\right\},\left\{b_{n}\right\}$ for which (1) does not hold, i.e. for which

$$
\text { (9) either } \varlimsup_{n \rightarrow \infty} \frac{b_{1}+\ldots+b_{n}}{a_{1}+\ldots+a_{n}}=\infty \text {, or } \varliminf_{n \rightarrow \infty} \frac{b_{1}+\ldots+b_{n}}{a_{1}+\ldots+a_{n}}=0 \text {, }
$$

we can choose $\left\{\varepsilon_{n}\right\}$ so that $\varepsilon_{n} \searrow 0$ and one of the two series $\left(\mathrm{A}^{\prime}\right),\left(\mathrm{B}^{\prime}\right)$ converges while the other diverges. Suppose, for instance, that the first alternative of (9) obtains. Then, given $k>1$, we can successively define positive integers $1=n_{0}, n_{1}, n_{2}, \ldots$ so that $n_{r}$ is the least integer greater than $n_{r-1}$ for which

$$
\frac{b_{1}+\ldots+b_{n_{r}}}{a_{1}+\ldots+a_{n_{r}}}>k^{r}, \quad r \geqslant 1 .
$$

If now we define $\left\{\varepsilon_{n}\right\}$ as follows:

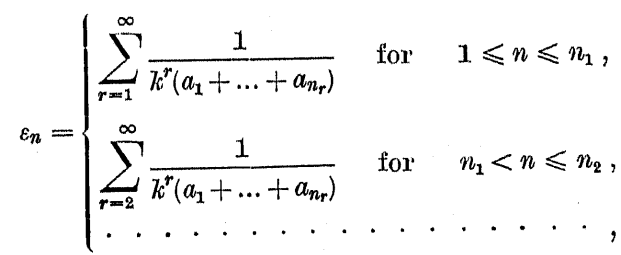


it is clear that the series $\left(\mathbf{A}^{\prime}\right),\left(\mathbf{B}^{\prime}\right)$ are:

$$
\begin{gathered}
\sum_{r=1}^{\infty}\left(a_{1}+\ldots+a_{n_{r}}\right)\left(\varepsilon_{n_{r}}-\varepsilon_{n_{r}+1}\right)=\frac{1}{k}+\frac{1}{k^{2}}+\ldots<\infty, \\
\sum_{r=1}^{\infty}\left(b_{1}+\ldots+b_{n_{r}}\right)\left(\varepsilon_{n_{r}}-\varepsilon_{n_{r}+1}\right)>1+1+\ldots=\infty .
\end{gathered}
$$

When the second alternative of (9) obtains, we interchange $\left\{a_{n}\right\}$ and $\left\{b_{n}\right\}$ in the above argument, so that finally, under the first (or second) alternative of (9), there is an $\left\{\varepsilon_{n}\right\}$ such that $\varepsilon_{n} \searrow 0$ and $\left(\mathbf{A}^{\prime}\right)$ converges (or diverges) while $\left(\mathbf{B}^{\prime}\right)$ diverges (or converges). This is what we set out to prove. The preceeding proof shows that we can separate Theorem I into two parts as follows:

THEOREM $I^{\prime}$. For every monotonic decreasing null sequence $\left\{\varepsilon_{n}\right\}$, the convergence of series (B) of Theorem I follows from that of series (A) if and only if

$$
\varlimsup_{n \rightarrow \infty} \frac{b_{1}+\ldots+b_{n}}{a_{1}+\ldots+a_{n}}<\infty .
$$

THEOREM I'. For every monotonic decreasing null sequence $\left\{\varepsilon_{n}\right\}$, the divergence of series (B) of Theorem I follows from that of series (A) if and only if

$$
\varliminf_{n \rightarrow \infty} \frac{b_{1}+\ldots+b_{n}}{a_{1}+\ldots+a_{n}}>0 .
$$

Deduction of Theorem II from Theorem I. $f(n)$ being subject to (2), in the light of Remarks 1,2 at the end of $\$ 2$, we can choose $\varepsilon_{n}=f(n) / n^{\beta}$, where $\beta>a$, in Theorem I. We can further choose, in Theorem I

$$
a_{n}=n^{\beta}, \quad b_{n}=\left\{\begin{array}{ccc}
\left(\lambda_{m}-\lambda_{m-1}\right) \lambda_{n}^{\beta} & \text { if } & n=\lambda_{m}, \\
0 & \text { if } & n \neq \lambda_{m}
\end{array}\right.
$$

and prove Theorem II by combining statements (14), (15), (16) below where $L=\varlimsup \overline{\lim } \lambda_{n} / \lambda_{n-1}$.

Firstly, whether $L$ is finite or not, (11) gives, for $\lambda_{m-1}<n \leqslant \lambda_{m}$,

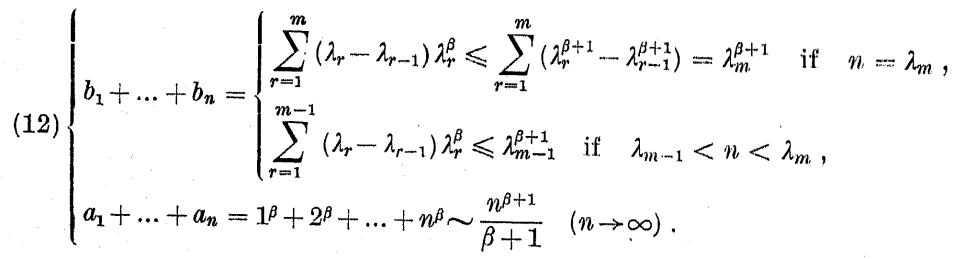

Hence

$$
\varlimsup_{n \rightarrow \infty} \frac{b_{1}+\ldots+b_{n}}{a_{1}+\ldots+a_{n}} \leqslant \beta+1,
$$

and Theorem I' above shows that,

(14) whether $L<\infty$ or $L=\infty$,

$$
\sum_{n=1}^{\infty} f(n)<\infty \text { ensures } \sum_{n=1}^{\infty}\left(\lambda_{n}-\lambda_{n-1}\right) f\left(\lambda_{n}\right)<\infty .
$$

Secondly, when $L=\infty$, we let $m \rightarrow \infty$ through a sequence of values for which $\lambda_{m} / \lambda_{m-1} \rightarrow \infty$ and, taking $n=\lambda_{m}-1$ in (12), we obtain

$$
\lim _{m \rightarrow \infty} \frac{b_{1}+\ldots+b_{\lambda_{m-1}}}{a_{1}+\ldots+a_{\lambda_{m-1}}}=0
$$

which violates condition $\left(1^{\prime \prime}\right)$ of the last Theorem $I^{\prime \prime}$ and shows that,

(15) when $L=\infty, \sum_{n=1}^{\infty} f(n)=\infty$ cannot always ensure $\sum_{n=1}^{\infty}\left(\lambda_{n}-\lambda_{n-1}\right) f\left(\lambda_{n}\right)=\infty$.

Lastly, when $L<\infty$ and $\lambda_{m-1}<n \leqslant \lambda_{m}, n \rightarrow \infty$, we get from (12):

i.e.

$$
\begin{gathered}
\frac{b_{1}+\ldots+b_{n}}{a_{1}+\ldots+a_{n}}>\frac{\sum_{r=1}^{m-1}\left(\lambda_{r}-\lambda_{r-1}\right) \lambda_{r}^{\beta}}{1^{\beta}+2^{\beta}+\ldots+\lambda_{m}^{\beta}}>\frac{\int_{\lambda_{0}}^{\lambda_{m-1}} x^{\beta} d x}{1^{\beta}+\ldots+\lambda_{m}^{\beta}} \sim\left(\frac{\lambda_{m-1}}{\lambda_{m}}\right)^{\beta+1}, \\
\varliminf_{n \rightarrow \infty} \frac{b_{1}+\ldots+b_{n}}{a_{1}+\ldots+a_{n}} \geqslant L^{-(\beta+1)} .
\end{gathered}
$$

And so Theorem $\mathrm{I}^{\prime \prime}$ shows that,

(16) when $L<\infty, \sum_{n=1}^{\infty} f(n)=\infty$ ensures $\sum_{n=1}^{\infty}\left(\lambda_{n}-\lambda_{n-1}\right) f\left(\lambda_{n}\right)=\infty$.

This completes the proof of Theorem Ir.

Proof of Theorem III. The convergence (or divergence) of series (A), under condition (4), implies the convergence (or divergence to $\pm \infty$ ) of series $(U)$ in consequence of Lemma 3 (or Lemma 4). After this it is obvious that the convergence (or non-convergence) of (U), under (4), implies the convergence (or divergence) of (A), the non-convergence of (U) being necessarily divergence to $\pm \infty$.

Remarks on Theorem III.

(i) Suppose that one of the limits in (4) is non-finite, e.g.

$$
\varlimsup_{n \rightarrow \infty} \frac{u_{1}+\ldots+u_{n}}{a_{1}+\ldots+a_{n}}=\infty .
$$

$\left(9^{\prime}\right)$ is simply the first alternative of (9) with $b_{n}$ replaced by $u_{n}$; hence, exactly as in the proof of Theorem I, we can first define by (10) a seAnnales Polonicl Mathematicl $\mathrm{xI}$ 
quence $\left\{\varepsilon_{n}\right\}$ in which $\varepsilon_{n} \searrow 0$ and then a series $\left(\mathrm{A}^{\prime}\right)$ which converges and secures, by Lemma 2, the convergence of series (A) as defined in Theorems I, III. Also, series ( $\left.\mathrm{U}^{\prime}\right)$, obtained with $u_{n}$ instead of $b_{n}$ in series $\left(\mathrm{B}^{\prime}\right)$ of the proof of Theorem I, diverges to $\infty$ like series $\left(\mathrm{B}^{\prime}\right)$; and

$$
u_{1}+\ldots+u_{n_{r}}>k^{r}\left(a_{1}+\ldots+a_{n_{r}}\right) \rightarrow \infty \quad \text { as } \quad r \rightarrow \infty .
$$

Hence identity (6), with $a_{n}$ replaced by $u_{n}$, shows that

$$
\left(u_{1} \varepsilon_{1}+\ldots+u_{n_{1}} \varepsilon_{n_{1}}\right)+\left(u_{n_{1}+1} \varepsilon_{n_{1}+1}+\ldots+u_{n_{2}} \varepsilon_{n_{2}}\right)+\ldots=\infty,
$$

or that series (U) as defined in Theorem III cannot converge.

Combining the argument used above with Lemma 3 , we get the following result supplementary to Theorem IIr.

THEOREM III'. Let $\left\{a_{n}\right\},\left\{u_{n}\right\}$ be given real sequences of which the first is positive. Then, for every monotonic decreasing null sequence $\left\{\varepsilon_{n}\right\}$, the convergence of series $(\mathrm{U})$ as defined in Theorem III follows from that of series (A) as defined there, if and onty if

(iia) Suppose that

$$
\varlimsup_{n \rightarrow \infty} \frac{\left|u_{1}+\ldots+u_{n}\right|}{a_{1}+\ldots+a_{n}}<\infty
$$

$$
\lim _{n \rightarrow \infty}\left|u_{1}+\ldots+u_{n}\right|=\infty, \quad \lim _{n \rightarrow \infty} \frac{\left|u_{1}+\ldots+u_{n}\right|}{a_{1}+\ldots+a_{n}}=0 .
$$

Then, given $k>1$, we can successively determine positive integers $1=n_{0}, n_{1}, n_{2}, \ldots$ so that $n_{r}$ is the least integer greater than $n_{r-1}(r \geqslant 1)$ for which

$$
\begin{aligned}
\left|u_{1}+\ldots+u_{n_{1}}\right|>0, & \frac{\left|u_{1}+\ldots+u_{n_{1}}\right|}{a_{1}+\ldots+a_{n_{1}}}<\frac{1}{k}, \\
\left|u_{1}+\ldots+u_{n_{r}}\right|>\left|u_{1}+\ldots+u_{n_{r-1}}\right|, & \frac{\left|u_{1}+\ldots+u_{n_{r}}\right|}{a_{1}+\ldots+a_{n_{r}}}<\frac{1}{k^{r}} \quad(r \geqslant 2) .
\end{aligned}
$$

Next we can define a monotonic decreasing null sequence $\left\{\varepsilon_{n}\right\}$ in terms of $\left\{\left|u_{1}+\ldots+u_{n}\right|\right\}$, exactly as we defined $\left\{\varepsilon_{n}\right\}$ in terms of $\left\{a_{1}+\ldots+a_{n}\right\}$ in (10). For this $\left\{\varepsilon_{n}\right\}$, series $\left(A^{\prime}\right)$ as defined in the proof of theorem $I$ is divergent, like the series $\left(B^{\prime}\right)$ in that proof, and so series $(A)$ as defined in Theorems I, III is also divergent by Lemma 2. Furthermore,

$$
\begin{aligned}
\sum_{n=1}^{\infty}\left|u_{1}+\ldots+u_{n}\right|\left(\varepsilon_{n}-\varepsilon_{n+1}\right) & =\sum_{r=1}^{\infty}\left|u_{1}+\ldots+u_{n_{r}}\right|\left(\varepsilon_{n_{r}}-\varepsilon_{n_{r}+1}\right)=\sum_{r=1}^{\infty} \frac{1}{k^{r}}, \\
\left|u_{1}+\ldots+u_{n_{r}}\right| \varepsilon_{n_{r}}<\frac{1}{k^{r-1}(k-1)} \rightarrow 0 \quad \text { as } \quad r \rightarrow \infty . &
\end{aligned}
$$

Consequently, by (6) with $a_{n}$ replaced by $u_{n}$,

$$
\left(u_{1} \varepsilon_{1}+\ldots+u_{n_{1}} \varepsilon_{n_{1}}\right)+\left(u_{n_{1}+1} \varepsilon_{n_{1}+1}+\ldots+u_{n_{2}} \varepsilon_{n_{2}}\right)+\ldots \text { is convergent, }
$$

and hence series (U) as defined in Theorem III cannot be divergent to $\pm \infty$. (iib) Suppose that

$$
\left(9^{\prime \prime} \mathrm{b}\right) \quad \quad \quad \lim _{n \rightarrow \infty}\left|u_{1}+\ldots+u_{n}\right|<\infty, \quad \sum_{n=1}^{\infty} a_{n}=\infty,
$$

with the implication that the lower limit in $\left(9^{\prime \prime} a\right)$ is again zero. From the first condition of $\left(9^{\prime \prime} \mathrm{b}\right)$, we can find a subsequence $\left\{m_{n}\right\}$ of $\{n\}$ and a number $K>0$ such that

$$
\left|u_{1}+u_{2}+\ldots+u_{m_{n}}\right|<K
$$

while, from the second condition of $\left(9^{\prime \prime} \mathrm{b}\right)$, we can choose in succession positive integers $n_{r}(r=1,2, \ldots)$ belonging to $\left\{m_{n}\right\}$ such that $n_{r}$ is the least integer greater than $n_{r-1}\left(r \geqslant 1, n_{0}=1\right)$ which makes, for a given $k>1$,

If then $\left\{\varepsilon_{n}\right\}$ is defined:

$$
a_{1}+a_{2}+\ldots+a_{n_{r}}>K k^{r} \text {. }
$$

$$
\varepsilon_{n}=\left\{\begin{array}{lll}
\sum_{r=1}^{\infty} \frac{1}{k^{r}} & \text { for } & 1 \leqslant n \leqslant n_{1}, \\
\sum_{r=2}^{\infty} \frac{1}{k^{r}} & \text { for } & n_{1}<n \leqslant n_{2}, \\
\ldots \ldots \ldots \ldots & \ldots \ldots \ldots
\end{array}\right.
$$

it is easy to prove as in (iia), in regard to series (A) and (U) defined as in Theorem III, that the first is divergent and the second is not so either to $+\infty$ or to $-\infty$.

(iia) and (iib) above cover all the cases in which the lower limit in $\left(9^{\prime \prime} \mathrm{a}\right)$ is zero and the divergence of $\Sigma a_{n}$ is assumed (either explicitly or implicitly) as a necessary pre-condition for the divergence of series (A) as defined in Theorem III. Hence (iia) and (iib), in conjunction with Lemma 4, establish the following complement to Theorem III'.

THEOREM III". Let $\left\{a_{n}\right\},\left\{u_{n}\right\}$ be given real sequences of which the first is positive. Then, for every monotonic decreasing null sequence $\left\{\varepsilon_{n}\right\}$, the divergence to $\pm \infty$ of series (U) as defined in Theorem III follows from the divergence of series (A) as defined there, if and only if

$$
\varliminf_{n \rightarrow \infty} \frac{\left|u_{1}+\ldots+u_{n}\right|}{a_{1}+\ldots+a_{n}}>0 .
$$

Deduction of Theorem IV from Theorem III. We choose, in Theorem III,

$$
\varepsilon_{n}=f\left(\lambda_{n}\right), \quad a_{n}=\lambda_{n}-\lambda_{n-1}, \quad b_{n}=\mu_{n}-\mu_{n-1},
$$

so that (4) takes the form (5), and appeal to the case $a=0$ of Theorem II. An additional theorem may be stated in conclusion since Corollary $\mathrm{V}$ following this theorem presents an interesting complement to Theorem II. 
THEOREM V. (a) If $\left\{a_{n}\right\},\left\{\varepsilon_{n}\right\}$ are positive sequences such that $\varepsilon_{n} \searrow 0$, POLONICI MATHEMATICI XI (1961)

$\sum_{n=1}^{\infty} a_{n} \varepsilon_{n}$ converges, then $\sum_{n=1}^{\infty}(-1)^{n-1}\left(a_{1}+\ldots+a_{n}\right) \varepsilon_{n}$ converges.

(b) If $\left\{b_{n}\right\}$ is another positive sequence such that

$$
\varlimsup_{n \rightarrow \infty} \frac{b_{1}+\ldots+b_{n}}{a_{1}+\ldots+a_{n}}<\infty
$$

then $\sum_{n=1}^{\infty}(-1)^{n-1}\left(b_{1}+\ldots+b_{n}\right) \varepsilon_{n}$ also converges.

Proof. (a) follows from Lemma 3 with $u_{n}=(-1)^{n-1}\left(a_{1}+\ldots+a_{1 b}\right)$

(b) follows from (a) since $\sum_{n-1}^{\infty} b_{n} \varepsilon_{n}$ is convergent by Lemma 3 again.

Theorem $\mathrm{V}$ gives rise to the following result, proved by Th. Kaluza ([2], Satz 23) by a different method in the particular case $\lambda_{n}=n$.

CoRoLlary. If $\beta \geqslant 0$ and $\left\{f(n) / n^{\beta}\right\}$ is ultimately positive monotonic decreasing, or equivalently $\{f(n)\}$ is positive quasi-monotonic decreasing, and if $\sum_{n=1}^{\infty} f(n)$ is convergent, $\left\{\lambda_{n}\right\}$ is a sequence of positive integer's monotonic increasing and unbounded, then $\sum_{n=1}^{\infty}(-1)^{n-1} \lambda_{n} f\left(\lambda_{n}\right)$ is convergent.

Pro of. Recalling Remark 1 at the end of $\$ 2$, we have only to choose, in Theorem $\nabla$ as modified by that remark,

$$
\varepsilon_{n}=\frac{f(n)}{n^{\beta}}, \quad a_{n}=n^{\beta}, \quad b_{n}=\left\{\begin{array}{ccc}
\lambda_{m}^{\beta+1}-\lambda_{m-1}^{\beta+1} & \text { if } & n=\lambda_{m}, \\
0 & \text { if } & n \neq \lambda_{m} .
\end{array}\right.
$$

This choice is justified since, arguing as we did with (11), we find that (13) holds for the above $\left\{b_{n}\right\}$ as well as for the $\left\{b_{n}\right\}$ in (11).

\section{References}

[1] A. Alexiewioz, On Canchy's condensation theorem, Studia Math. 16 (1957), p. $80-85$.

[2] Th. Kaluza, Struktur und Eigenschaften mehrfach monotoner Folgen, Math. Z. 26 (1927), p. $345-364$

[3] C. T. Rajagopal, On some limit theorems, Amer. J. Math. 70 (1948), p. 157-166 and p. 908

[4] O. Szász, Collected mathematioal papers, Cinoinnati 1955.

RAMANUJAN INSTITUTE OF MATHEMATICS, MADRAS, INDIA

Reģu par la Rédaction le 10.9. 1959

\section{Perturbations non linéaires qui n'augmentent pas la croissance maximale des intégrales}

\author{
par Z. SzMyDT (Kraków)
}

1. Envisageons le système d'équations différentielles linéaires, écrit sous la forme vectorielle

$$
\frac{d x}{d t}=A(t): c
$$

et le système perturbé

$$
\frac{d y}{d t}=A(t) y+f(t, y) .
$$

On suppose que $A(t)$ est une matrice carrée dont les éléments $a_{i j}(t)$ sont des fonctions de $t$, continues pour $t \geqslant 0$.

Soit $X(t)$ la matrice vérifiant les conditions

$$
\frac{d X}{d t}=A(t) X, \quad X(0)=I \quad(I \text { matrice unité }) .
$$

Nous l'appellerons matrice fondamentale du système (1).

Soient $\|x\|$ et $\|X\|$ les normes du vecteur $x=\left(x_{1}, \ldots, x_{n}\right)$ et de la matrice $X=\left(x_{i j}\right)$, données respectivement par les formules

$$
\|x\|=\sum_{i}\left|x_{i}\right|, \quad\|X\|=\sum_{i, j}\left|x_{i j}\right| .
$$

Dans cette note nous allons considérer le problème suivant.

PROBLìne Q. Correspond-il à chaque intégrale $y(t)$ du système (2) au moins une intégrale $x(t)$ du système (1) telle que

$$
y(t)=x(t)+o(\|X(t)\|) \text { ? }
$$

Dans le cas où le problème $\mathbf{Q}$ admet une réponse affirmative, chaque intégrale $y(t)$ du système (2) vérifie la relation $\left(^{1}\right)$

$$
y(t)=O(\|X(t)\|)
$$

(1) Cette relation a été étudiée dans [2]. Nous reviendrons sur ce sujet dans le emme 2 et dans la remarque 1 de cette note. 